\title{
Neoadjuvant Endocrine Therapy as an Alternative to Neoadjuvant Chemotherapy Among Hormone Receptor- Positive Breast Cancer Patients: Pathologic and Surgical Outcomes
}

\author{
Lifen Cao, MD, PhD ${ }^{1,2}$, Kavin Sugumar, $\mathrm{MD}^{1,2}$, Eleanor Keller, $\mathrm{BS}^{2,3}$, Pamela $\mathrm{Li}$, $\mathrm{MD}^{1,2}$, \\ Lisa Rock, MD ${ }^{1,2}$, Ashley Simpson, $\mathrm{DO}^{1,2}$, Mary Freyvogel, $\mathrm{DO}^{1,2}$, Alberto J. Montero, MD, $\mathrm{MBA}^{4}$, \\ Robert Shenk, MD, FACS ${ }^{1,2}$, and Megan E. Miller, MD, FACS ${ }^{1,2}$ \\ ${ }^{1}$ Division of Surgical Oncology, Department of Surgery, University Hospitals Cleveland Medical Center, Case Western \\ Reserve University School of Medicine, Cleveland, OH; ${ }^{2}$ University Hospitals Research in Surgical Outcomes and \\ Effectiveness (UH-RISES), Cleveland, $\mathrm{OH} ;{ }^{3} \mathrm{Case}$ Western Reserve University School of Medicine, Cleveland, OH; \\ ${ }^{4}$ Division of Hematology and Oncology, Department of Medicine, University Hospitals Cleveland Medical Center, Case \\ Western Reserve University School of Medicine, Cleveland, $\mathrm{OH}$
}

\begin{abstract}
Background. Neoadjuvant chemotherapy (NCT) is considered more effective in downstaging hormone receptorpositive $(\mathrm{HR}+)$ breast cancer than neoadjuvant endocrine therapy (NET), particularly in node-positive disease. This study compared breast and axillary response and survival after NCT and NET in HR+ breast cancer.

Methods. Based on American College of Surgeons Oncology Group (ACOSOG) Z1031 criteria, women age 50 years or older with cT2-4 HR+ breast cancer who underwent NET or NCT and surgery were identified in the National Cancer Database 2010-2016. Chi-square and logistic regression analysis determined differences between the NCT and NET groups and therapy response, including downstaging and pathologic complete response (pCR, ypT0/is and ypN0).
\end{abstract}

Presented in an e-Poster format at the American Society of Breast Surgeons International Conference on Breast Cancer Care, Virtual Meeting, 29 April to 2 May 2021

(C) Society of Surgical Oncology 2021

First Received: 22 April 2021

Accepted: 22 June 2021;

Published Online: 3 August 2021

M. E. Miller, MD, FACS

e-mail: Megan.Miller6@UHhospitals.org
Results. Of 19,829 patients, 14,025 (70.7\%) received NCT and $5804(29.3 \%)$ received NET. The NET patients were older (mean age, 68.9 vs. $60.3 ; P<0.001$ ) and had greater comorbidity (1+ Charlson-Deyo score, $21 \%$ vs. $16 \% ; P<$ 0.001). Therapy achieved $\mathrm{T}$ downstaging (any) for $58 \%$ of the patients with NCT versus $40.5 \%$ of the patients with NET, and in-breast pCR was achieved for $9.3 \%$ of the NCT versus $1.3 \%$ of the NET patients $(P<0.001)$. Approximately half of the mastectomy procedures could have been potentially avoided for the patients with in-breast pCR (53.6\% of the NCT and $43.8 \%$ of the NET patients). For the $\mathrm{cN}+$ patients, $\mathrm{N}$ downstaging (any) was $29 \%$ for the NCT patients versus $18.3 \%$ for the NET patients $(P<$ 0.001 ), and nodal pCR was achieved for $20.3 \%$ of the NCT versus $13.5 \%$ of the NET patients $(P<0.001)$. Among those with nodal pCR, axillary lymph node dissection (ALND) still was performed for $56 \%$ of the patients after NCT and $45 \%$ of the patients after NET.

Conclusions. Although the response rates after NCT were higher, NET achieved both $\mathrm{T}$ and $\mathrm{N}$ downstaging and $\mathrm{pCR}$. Neoadjuvant endocrine therapy can be used to de-escalate surgery for patients who cannot tolerate NCT or when chemotherapy may not be effective based on genomic testing.

Neoadjuvant chemotherapy (NCT) has been used for several decades to downstage locally advanced and unresectable primary breast cancers to facilitate operative 
management. ${ }^{1}$ Although historically, neoadjuvant endocrine therapy (NET) often was used primarily for elderly or frail patients with substantial comorbidities and those unable to tolerate chemotherapy, ${ }^{2}$ it has been used increasingly as an alternative to NCT for other patients with hormone receptor positive $(\mathrm{HR}+)$ and human epidermal growth factor receptor 2-negative (HER2-) disease. This receptor subtype is less likely to respond to NCT than others, such as HER2 + and triple-negative disease, with response rates as low as $9-38 \% .^{3-5}$ For such patients, using NET to achieve downstaging may enable surgery and improve both oncologic outcomes and cosmesis.

More recently, due to the COVID-19 pandemic, NET has been used in luminal A breast cancer to enable treatment and to maximize patient safety during temporary delays in surgery. ${ }^{6,7}$ In fact, $42 \%(951 / 2246)$ of patients had bridging endocrine therapy before surgery during the initial UK "Alert Level 4" phase of the pandemic, primarily because of reduced operating room capacity but also due to comorbidity and increased risk of COVID-19 mortality. ${ }^{8}$

Evidence for NET utilization is supported by robust clinical trial data, which have shown preoperative endocrine treatment to be an effective option, with significantly lower toxicity than NCT. ${ }^{9-12}$ Rates of breast-conserving surgery (BCS) after NET in large operable or locally advanced estrogen receptor-positive $(\mathrm{ER}+)$ breast cancers range from 60 to $80 \%{ }^{13-15}$ In the American College of Surgeons Oncology Group (ACOSOG) Z1031 phase II NET trial, a $69 \%$ response rate was reported with NET for women who had clinical stage 2 or 3 ER+ breast cancer. Half of the randomized patients who required mastectomy at diagnosis successfully underwent BCS after NET. ${ }^{9}$ However, the current literature shows that NET still is not widely used for HR+ patients. ${ }^{9}$ It is unclear whether this relates to an assumption that NET is much less effective than NCT, to patient selection criteria for NET versus $\mathrm{NCT}$, or to provider and/or patient preference.

This study aimed to identify HR+/HER2 - patients likely to benefit from NET based on ACOSOG Z1031 criteria and to compare those who were treated with NET versus NCT. The primary outcomes included breast and axillary response, surgical treatment, and overall survival. The secondary outcomes were predictors of response to NET versus NCT and the relationship of therapy response to survival. We anticipate that our results will help clinicians more confidently use NET for appropriate patients and thereby improve oncologic outcomes.

\section{METHODS}

\section{Data Collection and Elements}

After Institutional Review Board exemption due to the de-identified data source, a retrospective cohort study using the National Cancer Database (NCDB) was performed. Jointly sponsored by the American College of Surgeons and the American Cancer Society, the NCDB is a clinical oncology database sourced from hospital registry data representing more than $70 \%$ of cases with newly diagnosed cancer nationwide and 1500 Commission on Cancer (CoC)-accredited facilities. Definitions of the database variables are available from the dictionary of the NCDB Participant Use Data File (http://ncdbpuf.facs.org).

The CoC's NCDB and the hospitals participating in the CoC NCDB are the source of the de-identified data used in this study. They have not verified and are not responsible for the statistical validity of the data analysis or the conclusions derived by the authors.

\section{Patient Cohort and Data Analysis}

Based on National Comprehensive Cancer Network (NCCN) criteria for NET use and the ACOSOG Z1031 trial, which included postmenopausal women with clinical stage T2-4c ER+ breast cancer, ${ }^{9,16}$ the NCDB was queried for female patients older than 50 years with primary clinical stage T2-4 HR+/HER2 - breast cancer treated from 2010 to 2016. Tumors were considered HR+ if they were either ER+ or progesterone receptor-positive (PR+). Patients with incomplete treatment or follow-up data were excluded. Staging was based on the tumor-node metastasis (TNM) classification in the American Joint Committee on Cancer (AJCC) seventh edition. ${ }^{17}$

Patient demographics (age, race, insurance status, education level, household income, Charlson-Deyo comorbidity score), facility information (location, type, distance, setting), tumor characteristics (size, histologic grade, clinical stage, pathologic stage, hormone receptor [HR] status), and treatment factors (surgery, chemotherapy, endocrine therapy, sequence of treatment) were collected. Axillary surgery type was classified into two subgroups based on the Regional Lymph Nodes Examined variable as follows: sentinel lymph node biopsy (SLNB), defined as five or fewer lymph nodes examined, and axillary lymph node dissection (ALND), defined as more than five lymph nodes examined. The Scope of Regional Lymph Node Surgery variable, which defines SLNB versus ALND based on the operative report but is available only after 2012, was used to confirm the type of axillary surgery performed.

The cohort was categorized as receiving NCT or NET based on time-to-treatment sequence, derived from the start 
date of therapy versus surgery. The duration of neoadjuvant therapy was not available for inclusion in the analysis. The primary outcomes were tumor $(\mathrm{T})$ and nodal $(\mathrm{N})$ downstaging, type of breast and axillary surgery, and overall survival. Downstaging was defined by a pathologic stage lower than the clinical stage. Pathologic complete pathologic response (pCR) was defined as pathologic stage 0 , analogous to Residual Cancer Burden (RCB) score of 0 and Chevallier classification grade 1 . Secondary outcomes were predictors of response to NET versus NCT and the relationship of therapy response to survival.

Factors associated with neoadjuvant therapy were analyzed using the Wilcoxon rank-sum test for continuous variables and chi-square for categorical data. Multivariate analysis identified patient and tumor characteristics associated with receipt of NCT versus NET based on variables significant in the univariate analysis, as well as those with clinical significance. Differences in overall survival between the NET and NCT groups were analyzed using Kaplan-Meier survival estimates and compared through the log-rank test. A separate analysis of overall survival for the cohort of patients who received both neoadjuvant and adjuvant systemic therapy also was performed. All statistical analyses were performed using Stata/MP, version 16.0 (Stata Corp LLC, College Station, TX, USA).

\section{RESULTS}

\section{Clinical and Demographic Details}

Of 19,829 patients with a primary diagnosis of $\mathrm{HR}+/$ HER2 - clinical stage T2-4 invasive breast cancer, 14,025 (70.7\%) received NCT and 5804 (29.3\%) received NET (Table 1). Compared with the NCT patients, the NET patients were older (mean age, 68.9 vs. $60.3 ; P<0.001$ ), were more frequently white $(85.1 \%$ vs. $79.7 \% ; P<0.001)$, and had greater comorbidity (1+ Charlson-Deyo Score, $20.9 \%$ vs. $16.2 \% ; P<0.001)$. The majority of the NCT patients were clinically lymph node-positive, whereas most of the NET patients presented with node-negative disease (67.0\% vs. $36.8 \% ; P<0.001)$. The NCT patients were more likely to have lymphovascular invasion (LVI) and poorly differentiated tumors.

Nearly half of the patients after NET underwent partial mastectomy (NET $47.9 \%$ vs. NCT $31.5 \% ; P<0.001$; Table 2), whereas bilateral mastectomy was performed more often after NCT than after NET $(19.8 \%$ vs. $7.8 \% ; P<$ 0.001 ). For axillary surgery type, the Scope of Regional Lymph Node Surgery variable yielded unknown or missing data for one fourth of the patients, compared with the Number of Regional Lymph Nodes Examined variable, for which only $2-3 \%$ of the data were unknown or missing
(Table S1). Therefore, the latter variable was used for subsequent analysis.

SLNB was performed more frequently in NET than NCT patients $(47.1 \%$ vs. $34.0 \% ; P<0.001)$, mirroring the clinical nodal status in the two groups. After surgery, the vast majority of the NCT patients $(86.6 \%, 12,150 / 14,025)$ received adjuvant endocrine therapy, whereas only one fourth of the NET patients $(25.8 \%, 1497 / 4308)$ received adjuvant chemotherapy. Adjuvant radiation was administered more often to the NCT patients than to the NET patients $(P<0.001)$

\section{Factors Associated with Receipt of Neoadjuvant Endocrine Therapy Versus Neoadjuvant Chemotherapy}

Multivariable logistic regression was performed to determine patient and clinical characteristics independently associated with receipt of NET versus NCT (Table 3). After accounting for demographic and oncologic factors, patients with advanced age (odds ratio [OR], 5.1; $P<$ $0.001)$ had greater comorbidity (OR, $1.8 ; P<0.001)$, and that those treated at academic/integrated centers were more likely to undergo NET (OR, 1.6; $P<0.001$ ). Conversely, patients with poorly differentiated or undifferentiated tumors, more advanced clinical $\mathrm{T}$ stage, and clinically node-positive disease were less likely to receive NET than NCT (all $P<0.001$ ).

\section{Summary of Pathologic Outcomes}

Of the 19,829 patients, $\mathrm{T}$ downstaging (any) was achieved for $58.3 \%$ of the NCT patients and $40.5 \%$ of the NET patients, with breast pCR (pathologic T0) for $9.3 \%$ of the NCT patients and $1.3 \%$ of the NET patients $(P<0.001$; Table 4). Among those with breast pCR, the NET patients underwent partial mastectomy more frequently (NET $56.2 \%$ vs. NCT $46.4 \%$ ). However, mastectomy procedures could have potentially been avoided for approximately half of both the NCT patients $(53.6 \%, 645 / 1204)$ and the NET patients $(43.8 \%, 32 / 73)$, who achieved a complete pathologic in-breast tumor response (Table 4).

When in-breast tumor response was examined by clinical T stage, NCT yielded greater downstaging than NET across all clinical stages $(P<0.001$; Table 5). However, approximately one third of the patients who presented with cT2 disease achieved pT1 status in both treatment groups. The less robust response to NET than NCT was particularly notable in the group with more advanced disease at presentation, yet nearly $60 \%$ of the NET patients with cT4 tumors benefited from neoadjuvant treatment with some degree of in-breast downstaging.

Of the 10,784 clinically lymph node-positive patients, $8869(82.2 \%)$ received NCT and $1915(17.8 \%)$ received 
TABLE 1 Demographic and clinical characteristics of hormone receptor-positive (HR+) cT2-4 breast cancer patients (NCDB 2010-2016)

\begin{tabular}{|c|c|c|c|c|c|}
\hline Characteristics & $n$ & $\begin{array}{l}\text { Neoadjuvant } \\
\text { chemotherapy } \\
(n=14,025) \\
\%\end{array}$ & $n$ & $\begin{array}{l}\text { Neoadjuvant } \\
\text { endocrine therapy } \\
(n=5804) \\
\%\end{array}$ & $P$ value \\
\hline Age (years) & & & & & $<0.001$ \\
\hline Mean & 60.3 & & 68.9 & & \\
\hline $50-59$ & 7160 & 51.05 & 1271 & 21.9 & \\
\hline $60-69$ & 5121 & 36.51 & 1882 & 32.43 & \\
\hline $70-79$ & 1562 & 11.14 & 1550 & 26.71 & \\
\hline $80-89$ & 174 & 1.24 & 954 & 16.44 & \\
\hline $90+$ & 8 & 0.06 & 147 & 2.53 & \\
\hline Race & & & & & $<0.001$ \\
\hline White & 11,085 & 79.72 & 4906 & 85.07 & \\
\hline Black & 2144 & 15.42 & 611 & 10.59 & \\
\hline Asian or other & 676 & 4.86 & 250 & 4.34 & \\
\hline Charlson-Deyo score & & & & & $<0.001$ \\
\hline 0 & 11,755 & 83.81 & 4591 & 79.1 & \\
\hline 1 & 1840 & 13.12 & 860 & 14.82 & \\
\hline $2+$ & 430 & 3.07 & 353 & 6.08 & \\
\hline Insurance & & & & & $<0.001$ \\
\hline Not insured & 504 & 3.59 & 151 & 2.60 & \\
\hline Private & 7877 & 56.16 & 1894 & 32.63 & \\
\hline Public & 5483 & 39.09 & 3683 & 63.46 & \\
\hline Unknown & 161 & 1.15 & 76 & 1.31 & \\
\hline Facility type & & & & & $<0.001$ \\
\hline Community cancer program & 1410 & 10.05 & 494 & 8.51 & \\
\hline Comprehensive community cancer program & 6148 & 43.84 & 2364 & 40.73 & \\
\hline Academic/research program & 4388 & 31.29 & 2046 & 35.25 & \\
\hline Integrated network cancer program & 2079 & 14.82 & 900 & 15.51 & \\
\hline Facility location & & & & & $<0.001$ \\
\hline New England & 572 & 4.08 & 354 & 6.1 & \\
\hline Middle Atlantic & 1712 & 12.21 & 721 & 12.42 & \\
\hline South Atlantic & 3114 & 22.2 & 1228 & 21.16 & \\
\hline East North Central & 2486 & 17.73 & 941 & 16.21 & \\
\hline East South Central & 954 & 6.8 & 418 & 7.2 & \\
\hline West North Central & 1112 & 7.93 & 510 & 8.79 & \\
\hline West South Central & 1641 & 11.7 & 517 & 8.91 & \\
\hline Mountain & 718 & 5.12 & 288 & 4.96 & \\
\hline Pacific & 1716 & 12.24 & 827 & 14.25 & \\
\hline Facility area & & & & & 0.384 \\
\hline Metro & 11,683 & 85.44 & 4866 & 86.12 & \\
\hline Urban & 1747 & 12.78 & 681 & 12.05 & \\
\hline Rural & 244 & 1.78 & 103 & 1.82 & \\
\hline Distance to the hospital (miles) & & & & & $<0.001$ \\
\hline 0 & 2 & 0.01 & 1 & 0.02 & \\
\hline $0.1-20$ & 10,493 & 74.9 & 4413 & 76.07 & \\
\hline $20.1-40$ & 2045 & 14.6 & 722 & 12.45 & \\
\hline $40.1-60$ & 607 & 4.33 & 229 & 3.95 & \\
\hline $60.1-\max$ & 862 & 6.15 & 436 & 7.52 & \\
\hline
\end{tabular}


TABLE 1 continued

\begin{tabular}{|c|c|c|c|c|c|}
\hline Characteristics & $n$ & $\begin{array}{l}\text { Neoadjuvant } \\
\text { chemotherapy } \\
(n=14,025) \\
\%\end{array}$ & $n$ & $\begin{array}{l}\text { Neoadjuvant } \\
\text { endocrine therapy } \\
(n=5804) \\
\%\end{array}$ & $P$ value \\
\hline Clinical T stage & & & & & $<0.001$ \\
\hline 2 & 7166 & 51.09 & 3786 & 65.23 & \\
\hline 3 & 3614 & 25.77 & 1145 & 19.73 & \\
\hline 4 & 3245 & 23.14 & 873 & 15.04 & \\
\hline Clinical $N$ stage & & & & & $<0.001$ \\
\hline 0 & 5156 & 36.76 & 3889 & 67.01 & \\
\hline $1+$ & 8673 & 61.84 & 1795 & 30.93 & \\
\hline Unknown & 196 & 1.40 & 120 & 2.07 & \\
\hline Histology & & & & & $<0.001$ \\
\hline Ductal carcinoma & 10,508 & 74.92 & 3547 & 61.11 & \\
\hline Lobular and other invasive & 2858 & 20.38 & 1992 & 34.32 & \\
\hline Other & 659 & 4.7 & 265 & 4.57 & \\
\hline Grade & & & & & $<0.001$ \\
\hline Well-differentiated & 1136 & 8.10 & 1298 & 22.36 & \\
\hline Moderately differentiated & 5948 & 42.41 & 3100 & 53.41 & \\
\hline Poorly differentiated & 5732 & 40.87 & 1037 & 17.87 & \\
\hline Undifferentiated & 34 & 0.24 & 4 & 0.07 & \\
\hline Unknown & 1175 & 8.38 & 365 & 6.29 & \\
\hline Lymphovascular invasion & & & & & $<0.001$ \\
\hline Present & 4227 & 30.14 & 1320 & 22.74 & \\
\hline Not present & 6509 & 46.41 & 3363 & 57.94 & \\
\hline Unknown & 3289 & 23.45 & 1121 & 19.31 & \\
\hline$E R^{a}$ & & & & & $<0.001$ \\
\hline Positive & 13,478 & 96.11 & 5790 & 99.76 & \\
\hline Negative & 546 & 3.89 & 13 & 0.22 & \\
\hline Unknown & 1 & 0.01 & 1 & 0.02 & \\
\hline$P R^{a}$ & & & & & $<0.001$ \\
\hline Positive & 10,852 & 77.38 & 5061 & 87.20 & \\
\hline Negative & 3139 & 22.38 & 729 & 12.56 & \\
\hline Unknown & 34 & 0.24 & 14 & 0.24 & \\
\hline
\end{tabular}

Unknowns were excluded from the analysis

$N C D B$ National Cancer Database, $T$ tumor, $N$ nodal, $E R$ estrogen receptor, $P R$ progesterone receptor

${ }^{a}$ Tumors were considered hormone receptor-positive if either ER or PR was positive

NET. Therapy achieved $\mathrm{N}$ downstaging (any) for $29 \%$ of the NCT patients and $18.3 \%$ of the NET patients $(P<$ 0.001 ; Table 6). Nodal pCR was noted in $20.3 \%$ of the patients after NCT and $13.5 \%$ of the patients after NET (pathologic N0; $P<0.001$ ). ALND instead of SLNB still was performed in nearly half of both treatment groups despite nodal pCR (Table 6). However, there was an increase in SLNB use for the $\mathrm{cN}+$ patients over the study period, from $15.7 \%$ in 2010 to $24 \%$ in $2016(P<0.001)$.
Response to neoadjuvant therapy also was assessed for older patients and those with greater comorbidity at diagnosis (Table S2), because these groups have historically been prescribed NET rather than NCT. For the patients age 70 years or older and those with Charlson-Deyo score 1+, the breast tumor downstaging and nodal downstaging rates closely mirrored those in the overall population, with NCT yielding a greater response than NET in both groups $(P<$ $0.001)$. 
TABLE 2 Surgical and adjuvant treatment for hormone receptor-positive (HR+) cT2-4 breast cancer patients (NCDB 2010-2016)

\begin{tabular}{|c|c|c|c|c|c|}
\hline Characteristics & $n$ & $\begin{array}{l}\text { Neoadjuvant chemotherapy }(n=14025) \\
\%\end{array}$ & $n$ & $\begin{array}{l}\text { Neoadjuvant endocrine therapy }(n=5804) \\
\%\end{array}$ & $P$ value \\
\hline Breast surgery type & & & & & $<0.001$ \\
\hline Partial mastectomy & 4420 & 31.52 & 2779 & 47.88 & \\
\hline Unilateral mastectomy & 6833 & 48.72 & 2570 & 44.28 & \\
\hline Bilateral mastectomy & 2772 & 19.76 & 455 & 7.84 & \\
\hline Axillary surgery type & & & & & $<0.001$ \\
\hline SLNB (1-5 lymph nodes) & 4771 & 34.02 & 2732 & 47.07 & \\
\hline ALND ( $>5$ lymph nodes) & 8203 & 58.49 & 2253 & 38.82 & \\
\hline No axillary surgery or unknown & 1051 & 7.49 & 819 & 14.11 & \\
\hline \multicolumn{6}{|l|}{ Adjuvant chemotherapy ${ }^{\mathrm{a}}$} \\
\hline Yes & NA & NA & 1497 & 25.79 & \\
\hline Unknown & 0 & 0 & 125 & 2.15 & \\
\hline \multicolumn{6}{|l|}{ Adjuvant endocrine therapy ${ }^{\mathrm{b}}$} \\
\hline Yes & 12,150 & 86.63 & NA & NA & \\
\hline Unknown & 262 & 1.87 & 0 & 0 & \\
\hline Radiation therapy & & & & & $<0.001$ \\
\hline No & 2771 & 19.76 & 2405 & 41.44 & \\
\hline Yes & 10,889 & 77.64 & 3222 & 55.51 & \\
\hline Unknown & 365 & 2.6 & 177 & 3.05 & \\
\hline
\end{tabular}

$N C D B$ National Cancer Database, $S L N B$ sentinel lymph node biopsy, $A L N D$ axillary lymph node dissection

${ }^{a}$ The sequence of treatment was used to determine whether neoadjuvant endocrine therapy (NET) patients received adjuvant chemotherapy.

${ }^{\mathrm{b}}$ The sequence of treatment was used to determine whether neoadjuvant chemotherapy (NCT) patients received adjuvant endocrine therapy.

Survival Analysis Using the Kaplan-Meier estimate, the patients who received NCT with complete or partial response had superior 5-year overall survival than the noresponse groups $(78.4 \%$ vs. $70.3 \%[P<0.001]$ for $\mathrm{T}$ response; $77.1 \%$ vs. $69.5 \%[P<0.001]$ for $\mathrm{N}$ response; Fig. 1). Similarly, the patients who received NET with complete or partial response also had an overall 5-year survival benefit compared with the no-response group (78.5\% vs. $67.4 \%[P<0.001]$ for $\mathrm{T}$ response; $73.7 \%$ vs. $63.4 \%[P=0.013]$ for $\mathrm{N}$ response).

Nearly $70 \%(13,647 / 19,829)$ of the entire cohort received both neoadjuvant and adjuvant systemic therapy, most frequently with NCT followed by adjuvant endocrine therapy. Overall survival was similar in the group that received NCT followed by adjuvant endocrine therapy and those treated with NET followed by adjuvant chemotherapy $(77.1 \%$ vs. $76.4 \%[P=0.697]$ at 5 years, Fig. 2).

\section{DISCUSSION}

Although NCT downstages breast cancer, NET currently is increasingly used not only for advanced $\mathrm{HR}+$ disease and patients who are not chemotherapy candidates, but also for HR+ breast cancer because of its known poor response to NCT. Consistent with historic trends, patients in our study who received NET were older with greater comorbidity, had low-grade tumors, low clinical $\mathrm{T}$ stage, and were node-negative. Although our finding of low pCR with NET was similar to that of prior studies reporting a pCR of only $1-2 \%$ for in-breast tumors and $11-13 \%$ for axillary disease, ${ }^{18,19}$ downstaging was achieved for approximately $40 \%$ of tumors and $20 \%$ of clinically positive lymph nodes in our study. Moreover, the patients who received NET with any response (complete or partial) had a survival benefit of similar magnitude to those downstaged with NCT, which may be even more clinically significant for the older and more comorbid patient population often selected for NET. Yet NET was still used significantly less frequently than NCT for the patients with HR+/HER2disease, consistent with prior literature demonstrating underutilization of NET. ${ }^{9}$

Underutilization of NET may be due to concerns regarding its effectiveness compared with NCT. A metaanalysis of 20 prospective, randomized clinical trials evaluating NET for patients with ER+ breast cancer showed similar response and BCS rates with aromatase inhibitor-based neoadjuvant endocrine monotherapy and combination neoadjuvant chemotherapy. ${ }^{20}$ However, other prior studies have demonstrated greater benefit with NCT than with NET in achieving pCR. A single-institution study 
TABLE 3 Multivariable logistic regressions for predictors of receipt of NET versus NCT in hormone receptor-positive $(\mathrm{HR}+)$ cT2-4 breast cancer patients (NCDB 2010-2016)

\begin{tabular}{|c|c|c|c|c|}
\hline \multirow{2}{*}{$\begin{array}{l}\text { NET versus NCT } \\
\text { Age } \geq 70 \text { versus } 50-69 \text { years }\end{array}$} & \multirow{2}{*}{$\begin{array}{l}\text { OR } \\
5.078\end{array}$} & \multicolumn{2}{|c|}{$95 \% \mathrm{CI}$} & \multirow{3}{*}{$\begin{array}{l}P \text { value } \\
<0.001\end{array}$} \\
\hline & & 4.562 & 5.652 & \\
\hline Race & & & & \\
\hline White & \multicolumn{3}{|c|}{ Reference } & \\
\hline African American & 0.738 & 0.646 & 0.844 & $<0.001$ \\
\hline Asian or others & 0.970 & 0.790 & 1.191 & 0.770 \\
\hline \multicolumn{5}{|l|}{ Charlson-Deyo score } \\
\hline 0 & \multicolumn{3}{|c|}{ Reference } & \\
\hline 1 & 1.081 & 0.957 & 1.221 & 0.212 \\
\hline $2+$ & 1.810 & 1.476 & 2.220 & $<0.001$ \\
\hline \multicolumn{5}{|l|}{ Facility type } \\
\hline Community & \multicolumn{3}{|c|}{ Reference } & \\
\hline Comprehensive & 1.129 & 0.967 & 1.318 & 0.124 \\
\hline Academic & 1.587 & 1.354 & 1.861 & $<0.001$ \\
\hline Integrated & 1.278 & 1.071 & 1.526 & 0.006 \\
\hline \multicolumn{5}{|l|}{ Insurance status } \\
\hline \multicolumn{5}{|l|}{ Not insured } \\
\hline Private insurance & 0.640 & 0.502 & 0.816 & $<0.001$ \\
\hline Public insurance & 0.981 & 0.769 & 1.253 & 0.880 \\
\hline \multicolumn{5}{|l|}{ Grade } \\
\hline Well-differentiated & \multicolumn{3}{|c|}{ Reference } & \\
\hline Moderately differentiated & 0.483 & 0.430 & 0.543 & $<0.001$ \\
\hline Poorly differentiated & 0.179 & 0.157 & 0.205 & $<0.001$ \\
\hline Undifferentiated & 0.116 & 0.031 & 0.426 & 0.001 \\
\hline Lymphovascular invasion & 1.035 & 0.942 & 1.138 & 0.473 \\
\hline \multicolumn{5}{|l|}{ Clinical T stage } \\
\hline 2 & \multicolumn{3}{|c|}{ Reference } & \\
\hline 3 & 0.642 & 0.577 & 0.714 & $<0.001$ \\
\hline 4 & 0.579 & 0.513 & 0.653 & $<0.001$ \\
\hline \multicolumn{5}{|l|}{ Clinical N stage } \\
\hline 0 & \multicolumn{3}{|c|}{ Reference } & \\
\hline 1 & 0.345 & 0.314 & 0.380 & $<0.001$ \\
\hline 2 & 0.325 & 0.271 & 0.389 & $<0.001$ \\
\hline 3 & 0.290 & 0.225 & 0.374 & $<0.001$ \\
\hline
\end{tabular}

NET neoadjuvant endocrine therapy, $N C T$ neoadjuvant chemotherapy, $N C D B$ National Cancer Database, $T$ tumor, $N$ nodal

of $127 \mathrm{HR}+/ \mathrm{HER} 2-$ cancers treated with NET and 338 HR+/HER2 - cancers treated with NCT reported higher breast pCR rates with NCT than with NET $(0 \%$ vs. $9 \% ; P=$ $0.035)$ but similar nodal pCR rates $(11 \%$ vs. $18 \% ; P=$ 0.37), although only four NET patients in this series achieved nodal pCR. ${ }^{18}$ Similarly, an NDCB analysis of stages 2 and $3 \mathrm{HR}+/ \mathrm{HER} 2-$ breast cancers treated in 2012-2015 showed a significantly lower overall pCR rate after NET than after NCT $(1.1 \%$ vs. $8.4 \% ; P<0.001)$, as well as a lower nodal pCR rate (NET $13.3 \%$ vs. NCT
$22.8 \% ; P<0.001) .{ }^{19}$ Our findings of breast $\mathrm{pCR}$ in $9.3 \%$ of the NCT and $1.3 \%$ of the NET patients and a nodal pCR of $20.3 \%$ for NCT versus $13.5 \%$ for NET (both $P<0.001$ ) confirm these previously described patterns. However, the effectiveness of neoadjuvant therapy is fortunately not limited to pCR only.

Breast tumor downstaging alone may facilitate BCS rather than mastectomy, and it logically follows that breast pCR provides an even greater likelihood of BCS. Our data show that although breast tumor downstaging was more robust after NCT, more than $50 \%$ of cT3 and $40 \%$ of cT4 lesions decreased to pT2 or less after NET, likely meeting the criteria for BCS. This compares favorably with BCS rates after NET of $77 \%$ and $46 \%$ reported in prior studies. ${ }^{9,18}$ Thus, although NCT may be more effective than NET in achieving pCR, the breast tumor downstaging achieved after NET can enable BCS, providing a clinical advantage via de-escalation of surgery.

In our study, nearly half of mastectomy procedures could have been potentially avoided for patients with breast pCR after NET, and BCS could have been used more frequently in the $40 \%$ of patients with any $\mathrm{T}$ downstaging. The NET patients age 70 or older and those with a Charlson-Deyo score of $1+$ had downstaging rates similar to those of the overall NET population (36.2\% and 38.9\%, respectively), suggesting that NET is a viable strategy for patients who may not be ideal candidates for chemotherapy or mastectomy. This represents an opportunity for multidisciplinary breast cancer care teams to tailor neoadjuvant therapy choice and surgery type to both individual patients and clinical response, acknowledging the risks, benefits, and response rates of each treatment approach.

Several randomized clinical trials and current national guidelines support the safety and efficacy of SLNB after NCT for clinically node-positive disease with favorable response, thereby decreasing the need for ALND. ${ }^{12,16,21,22}$ These recommendations can be reasonably extrapolated to NET, especially when methods to improve SLN identification are used. The initial concerns regarding SLNB after NCT included potential underlying changes to lymphatic drainage with tumor response to treatment and resulting fibrosis, and are similar with NET. However, using dualtracer mapping and retrieval of more than two sentinel lymph nodes resulted in a false-negative rate for SLNB lower than $10 \%$ after NCT and could be similarly used in the NET setting. ${ }^{21,22}$ Placing a clip at the time of lymph node biopsy and ensuring its removal with an intraoperative localization technique lowers the false-negative rate even further, and could also be considered after NET as with NCT. ${ }^{16,23}$

Despite these findings and recommendations, SLNB was underutilized in both groups (NCT and NET) of our study, especially for those who had a nodal pCR. This assessment 
TABLE 4 Breast tumor response to neoadjuvant therapy for HR+ cT2-4 breast cancer patients (NCDB 2010-2016)

\begin{tabular}{|c|c|c|c|c|c|}
\hline Characteristics & $n$ & $\begin{array}{l}\text { Neoadjuvant chemotherapy } \\
(n=14,025) \\
\%\end{array}$ & $n$ & $\begin{array}{l}\text { Neoadjuvant endocrine therapy } \\
(n=5804) \\
\%\end{array}$ & $P$ value \\
\hline \multicolumn{5}{|l|}{ Clinical T stage } & $<0.001$ \\
\hline 2 & 7166 & 51 & 3786 & 65 & \\
\hline 3 & 3614 & 26 & 1145 & 20 & \\
\hline 4 & 3245 & 23 & 873 & 15.04 & \\
\hline Total & 14,025 & 100 & 5804 & 100 & \\
\hline \multicolumn{5}{|l|}{ Pathologic T stage } & $<0.001$ \\
\hline 0 & 1204 & 9.28 & 73 & 1.33 & \\
\hline 1 & 3985 & 30.71 & 1383 & 25.25 & \\
\hline 2 & 4821 & 37.15 & 2768 & 50.54 & \\
\hline 3 & 1871 & 14.42 & 799 & 14.59 & \\
\hline 4 & 1096 & 8.45 & 454 & 8.29 & \\
\hline Total & 12,977 & 100 & 5477 & 100 & \\
\hline \multicolumn{5}{|l|}{ Tumor downstaging (any) } & $<0.001$ \\
\hline No & 5416 & 41.74 & 3257 & 59.47 & \\
\hline Yes & 7561 & 58.26 & 2220 & 40.53 & \\
\hline Total & 12,977 & 100 & 5477 & 100 & \\
\hline \multicolumn{5}{|c|}{ Breast surgery type for patients with complete in-breast tumor response } & 0.077 \\
\hline Partial mastectomy & 559 & 46.43 & 41 & 56.16 & \\
\hline Unilateral mastectomy & 385 & 31.98 & 24 & 32.88 & \\
\hline Bilateral mastectomy & 260 & 21.59 & 8 & 10.96 & \\
\hline Total & 1204 & 100 & 73 & 100 & \\
\hline
\end{tabular}

$N C D B$ National Cancer Database, $T$ tumor

was based on presumed use of SLNB with intraoperative frozen section for $\mathrm{cN}+$ disease after appropriate response to neoadjuvant therapy. We equated nodal $\mathrm{pCR}$ with negative frozen section, indicating that ALND was unnecessary. ${ }^{16,24}$ Although this practice is the current standard of care, it may not have been implemented at the start of the study period in 2010, potentially skewing our findings. When queried longitudinally, there was an increase in SLNB rate in later years for $\mathrm{cN}+$ patients. However, the ALND rate remained nearly $70 \%$ in 2016, and more than half of the patients with nodal pCR still underwent ALND. Greater awareness and use of the aforementioned evidence-based surgical techniques to reduce ALND after NCT may decrease ALND rates after NET as well.

As expected, patients with advanced disease and aggressive tumor features were more likely to undergo NCT than NET in our study. However, because NET also achieved $\mathrm{T}$ and $\mathrm{N}$ downstaging and because this approach may be more feasible for older patients and those with comorbidity, especially in the ongoing COVID-19 pandemic, it is necessary to identify which patients will benefit from NET versus NCT.
In recent years, there has been an increased reliance on biomarker assays to guide adjuvant systemic therapy decisions for women with early-stage HR+ breast cancer, such as Oncotype DX21 Recurrence Score, EndoPredict, and the Amsterdam 70 gene profile (MammaPrint). ${ }^{6}$ Unfortunately, data on the use of genomic assays in the NCDB is limited-only $10 \%$ of the patients in our study had documented Mammaprint or Oncotype results.

In clinical practice, shifting the use of genomic assays to core needle biopsy rather than surgical pathology could identify which tumors are likely to respond to NCT and thereby assist with neoadjuvant decision-making. In turn, this could potentially increase response rates to both NET and NCT by appropriately selecting patients for each therapeutic method. Predictive assays or scoring tools used at diagnosis also would facilitate more widespread use of NET with less morbidity than NCT. The reduction of toxicity including nausea, vomiting, hair loss, myelosuppression, and cognitive impairment seen with NET versus NCT may be particularly important for older patients with comorbidity already at greater risk of treatment-related complications. ${ }^{20,25}$ Furthermore, although tumors with strong ER expression are expected to respond well to endocrine therapy, the intensity of ER positivity does not 
TABLE 5 Breast tumor pathologic stage after neoadjuvant therapy stratified by clinical stage for hormone receptor-positive (HR+) cT2-4 breast cancer patients (NCDB 2010-2016)

\begin{tabular}{|c|c|c|c|c|c|}
\hline $\mathrm{T}$ stage & $n$ & $\begin{array}{l}\text { Neoadjuvant chemotherapy } \\
(n=14,025) \\
\%\end{array}$ & $n$ & $\begin{array}{l}\text { Neoadjuvant endocrine therapy } \\
(n=5804) \\
\%\end{array}$ & $P$ value \\
\hline Clinical T2 & 6673 & 100 & 3600 & 100 & $<0.001$ \\
\hline pT0 & 764 & 11.45 & 49 & 1.36 & \\
\hline pT1 & 2593 & 38.86 & 1156 & 32.11 & \\
\hline pT2 & 2904 & 43.52 & 2115 & 58.75 & \\
\hline pT3 & 345 & 5.17 & 225 & 6.25 & \\
\hline pT4 & 67 & 1 & 55 & 1.53 & \\
\hline Clinical T3 & 3356 & 100 & 1069 & 100 & $<0.001$ \\
\hline pT0 & 236 & 7.03 & 16 & 1.5 & \\
\hline pT1 & 794 & 23.66 & 138 & 12.91 & \\
\hline pT2 & 1173 & 34.95 & 408 & 38.17 & \\
\hline pT3 & 1071 & 31.91 & 463 & 43.31 & \\
\hline pT4 & 82 & 2.44 & 44 & 4.12 & \\
\hline Clinical T4 & 2948 & 100 & 808 & 100 & $<0.001$ \\
\hline pT0 & 204 & 6.92 & 8 & 0.99 & \\
\hline pT1 & 598 & 20.28 & 89 & 11.01 & \\
\hline pT2 & 744 & 25.24 & 245 & 30.32 & \\
\hline pT3 & 455 & 15.43 & 111 & 13.74 & \\
\hline pT4 & 947 & 32.12 & 355 & 43.94 & \\
\hline
\end{tabular}

$N C D B$ National Cancer Database

always correlate with response, and other parameters such as PR and Ki67 expression play a role. ${ }^{26-28}$ Thus, the development of more accurate predictive and prognostic tools may better guide the NCT versus NET decision as well as the choice of therapeutic medications (i.e., aromatase inhibitor vs. tamoxifen). In other patients, primary surgery may emerge as the treatment of choice.

Our results also address the potential bias against NET based on two assumptions: that NET will not avoid chemotherapy and that delayed adjuvant chemotherapy for patients likely to need chemotherapy after NET will have a negative impact on outcomes. In our study, only $25 \%$ of patients who received NET were also treated with adjuvant chemotherapy. Therefore, NET helped to avoid adjuvant chemotherapy in a proportion of patients who, based on clinical stage, likely would have been offered chemotherapy. Furthermore, overall survival was similar in those who underwent NET followed by adjuvant chemotherapy versus NCT followed by adjuvant endocrine therapy regardless of treatment sequence, negating the second assumption. This aligns with prior literature demonstrating improved survival of patients with $\mathrm{HR}+\mathrm{cN}+$ breast cancer who received both neoadjuvant and adjuvant systemic therapy. ${ }^{29}$ Although it was not possible to assess with NCDB data, downstaging with NET also may allow de-intensification of chemotherapy (e.g., from more toxic anthracycline-based regimens) even if it does not obviate the need for chemotherapy altogether.

Our study had several limitations relating to factors not captured in large retrospective cohorts such as the NCDB. The database does not include local regional recurrence or disease-free survival. Thus, our analysis of long-term outcomes was limited to overall survival. Similarly, granular details about NCT or NET agents, doses, combinations, toxicities, and decision-making are not included and therefore cannot be factored into analyses. The NCDB does not contain information about multifocal or multicentric disease, diagnostic imaging, or imaging response, all of which affect surgical planning. For example, extensive calcifications that remain after neoadjuvant therapy may prompt mastectomy based on known inconsistencies between $\mathrm{pCR}$ and imaging characteristics, and lack of nodal response evidenced by residual lymphadenopathy via exam or imaging still would indicate ALND. ${ }^{30}$

Furthermore, the NCDB lacks data on patient preference and surgical judgement. Thus, we relied on staging data to determine the feasibility of BCS versus mastectomy and SLNB versus ALND. Although our definition of SLNB 
TABLE 6 Axillary response to neoadjuvant therapy for hormone receptor-positive (HR+) cT2-4 breast cancer patients (NCDB 2010-2016)

\begin{tabular}{|c|c|c|c|c|c|}
\hline Characteristics & $n$ & $\begin{array}{l}\text { Neoadjuvant chemotherapy } \\
(n=8869) \\
\%\end{array}$ & $n$ & $\begin{array}{l}\text { Neoadjuvant endocrine therapy } \\
(n=1915) \\
\%\end{array}$ & $P$ value \\
\hline Clinical N stage & & & & & $<0.001$ \\
\hline 1 & 6401 & 73.8 & 1402 & 78.11 & \\
\hline 2 & 1423 & 16.41 & 273 & 15.21 & \\
\hline 3 & 849 & 9.79 & 120 & 6.69 & \\
\hline Total & 8673 & 100 & 1795 & 100 & \\
\hline Pathologic N stage & & & & & $<0.001$ \\
\hline 0 & 1667 & 20.33 & 231 & 13.53 & \\
\hline 1 & 3307 & 40.33 & 734 & 43 & \\
\hline 2 & 2186 & 26.66 & 471 & 27.59 & \\
\hline 3 & 1039 & 12.67 & 271 & 15.88 & \\
\hline Total & 8199 & 100 & 1707 & 100 & \\
\hline$N$ downstaging (any) & & & & & $<0.001$ \\
\hline No & 5700 & 71.05 & 1317 & 81.75 & \\
\hline Yes & 2322 & 28.95 & 294 & 18.25 & \\
\hline Total & 8022 & 100 & 1611 & 100 & \\
\hline \multicolumn{6}{|c|}{ Axillary surgery type for patients with complete nodal response } \\
\hline Lymph nodes examined & & & & & $=0.009$ \\
\hline SLNB (1-5) & 628 & 43.85 & 87 & 54.72 & \\
\hline $\operatorname{ALND}(\geq 6)$ & 804 & 56.15 & 72 & 45.28 & \\
\hline Total & 1432 & 100 & 159 & 100 & \\
\hline
\end{tabular}

$N C D B$ National Cancer Database, $N$ nodal, $S L N B$ sentinel lymph node biopsy, $A L N D$ axillary lymph node dissection

FIG. 1 Overall survival after neoadjuvant therapy for $\mathrm{HR}+$ cT2-4 breast cancer patients stratified by treatment response, NCDB 2010-2016. NCT neoadjuvant chemotherapy, NET neoadjuvant endocrine therapy
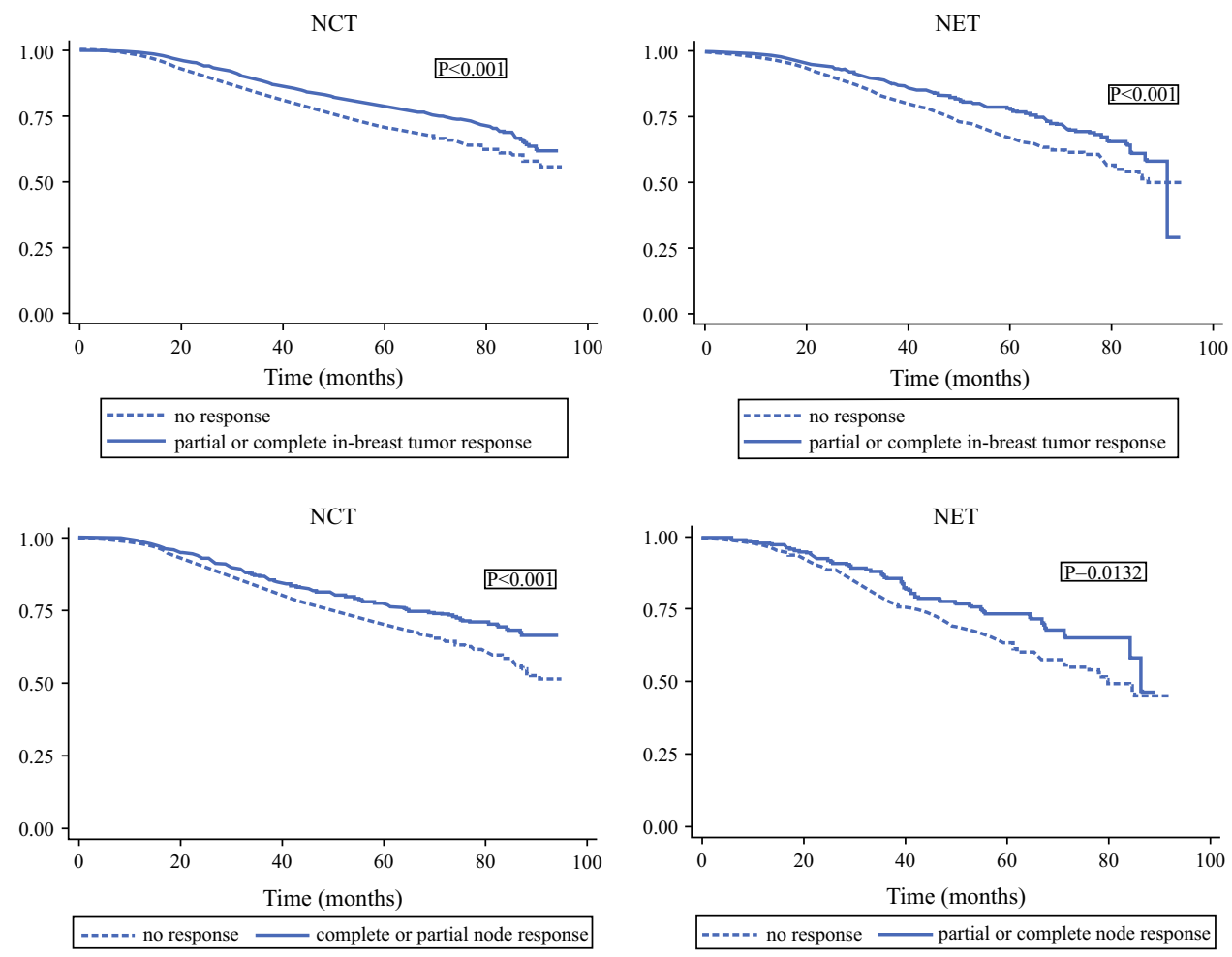


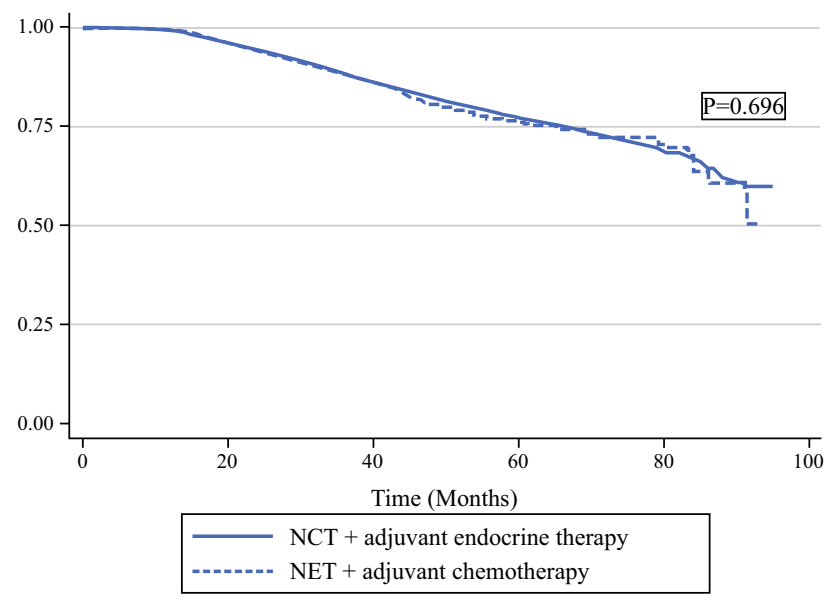

FIG. 2 Overall survival for patients after neoadjuvant therapy and adjuvant therapy for HR+ cT2-4 breast cancer, NCDB 2010-2016. $N C T$ neoadjuvant chemotherapy, NET neoadjuvant endocrine therapy

versus ALND was based on clinical consensus and mean number of nodes retrieved in large prospective studies, ${ }^{21,31-33}$ we readily acknowledge the variability in the number of nodes removed during SLNB versus ALNDalthough reassuringly, ALND rates were comparable between the two NCBD variables (Scope of Regional Lymph Node Surgery and Number of Regional Lymph Nodes Examined).

Due to the inherent limitations of retrospective research reliant on large de-identified datasets, our analysis intentionally excluded patients with missing clinical and followup information, which may have led to systematic exclusion bias. Additionally, effect size may have been falsely elevated when groups in such datasets were compared, yielding statistical significance without clinical relevance.

Because our study focused on postmenopausal women meeting the ASOCOG Z1031 criteria, the results may not be generalizable to other populations with breast cancer. Investigation of the benefit from NET for pre-menopausal women and for men with HR + disease will be an important area for further study.

\section{CONCLUSIONS}

Although the response rates for NCT are higher, NET achieves $\mathrm{T}$ and $\mathrm{N}$ downstaging and $\mathrm{pCR}$ in $\mathrm{HR}+$ patients. For patients with pCR, NET can be used to increase BCS rates and prevent ALND, especially with methods to ensure accurate SLNB. Multigene signature or future predictive assays will assist with decision-making in $\mathrm{HR}+$ disease by identifying which tumors will respond to chemotherapy, potentially allowing more NET use with less morbidity and increasing response rates with both NET and NCT for appropriately selected patients. Finally, improved survival for those who respond to NET should be factored into neoadjuvant treatment choice and could encourage wider use for patients who may not be candidates for NCT.

Supplementary Information The online version contains supplementary material available at https://doi.org/10.1245/s10434021-10459-3.

DISCLOSURE There are no conflicts of interest.

\section{REFERENCES}

1. Schick P, Goodstein J, Moor J, Butler J, Senter KL. Preoperative chemotherapy followed by mastectomy for locally advanced breast cancer. J Surg Oncol. 1983;22:278-82.

2. Network ACSCA. COVID-19 pandemic ongoing impact on cancer. Patients and Survivors Survey Findings Summary. 2020; 2020.

3. Coates AS, Colleoni M, Goldhirsch A. Is adjuvant chemotherapy useful for women with luminal a breast cancer? J Clin Oncol. 2012;30:1260-3.

4. Hayes DF. Targeting adjuvant chemotherapy: a good idea that needs to be proven! J Clin Oncol. 2012;30:1264-7.

5. Schott AF, Hayes DF. Defining the benefits of neoadjuvant chemotherapy for breast cancer. J Clin Oncol. 2012;30:1747-9.

6. Korde LA, Somerfield MR, Carey LA, et al. Neoadjuvant chemotherapy, endocrine therapy, and targeted therapy for breast cancer: ASCO guideline. J Clin Oncol. 2021;39:1485-505.

7. Park KU, Gregory M, Bazan J, et al. Neoadjuvant endocrine therapy use in early-stage breast cancer during the covid-19 pandemic. Breast Cancer Res Treat. 2021;188:249-58.

8. Dave RV, Kim B, Courtney A, et al. Breast cancer management pathways during the COVID-19 pandemic: outcomes from the UK "Alert Level 4" phase of the B-MaP-C study. Br J Cancer. 2021;124:1785-94.

9. Chiba A, Hoskin TL, Heins CN, Hunt KK, Habermann EB, Boughey JC. Trends in neoadjuvant endocrine therapy use and impact on rates of breast conservation in hormone receptor-positive breast cancer: a National Cancer Data Base study. Ann Surg Oncol. 2017;24:418-24.

10. Eiermann W, Paepke S, Appfelstaedt J, et al. Preoperative treatment of postmenopausal breast cancer patients with letrozole: a randomized double-blind multicenter study. Ann Oncol. 2001;12:1527-32.

11. Ellis MJ, Suman VJ, Hoog J, et al. Randomized phase II neoadjuvant comparison between letrozole, anastrozole, and exemestane for postmenopausal women with estrogen receptorrich stage 2 to 3 breast cancer: clinical and biomarker outcomes and predictive value of the baseline PAM50-based intrinsic subtype-ACOSOG Z1031. J Clin Oncol. 2011;29:2342-9.

12. Smith IE, Dowsett M, Ebbs SR, et al. Neoadjuvant treatment of postmenopausal breast cancer with anastrozole, tamoxifen, or both in combination: the immediate preoperative anastrozole, tamoxifen, or combined with tamoxifen (IMPACT) multicenter double-blind randomized trial. J Clin Oncol. 2005;23:5108-16.

13. Arthur LM, Turnbull AK, Khan LR, Dixon JM. Preoperative endocrine therapy. Curr Breast Cancer Rep. 2017;9:202-9.

14. Cain H, Macpherson IR, Beresford M, Pinder SE, Pong J, Dixon JM. Neoadjuvant therapy in early breast cancer: treatment considerations and common debates in practice. Clin Oncol $R$ Coll Radiol. 2017;29:642-52.

15. Sato N, Masuda N, Morimoto T, et al. Neoadjuvant endocrine therapy with exemestane followed by response-guided 
combination therapy with low-dose cyclophosphamide in postmenopausal patients with estrogen receptor-positive breast cancer: a multicenter, open-label, phase II study. Cancer Med. 2018;7:3044-56.

16. William J, Gradishar WJ, Aft R, Agnese D, Allison K, Blair SL. NCCN guidelines: breast cancer, version 6. J Natl Compr Cancer Netw. 2020;18:452-78.

17. Edge SB, Compton CC. The American Joint Committee on Cancer: the 7th edition of the AJCC cancer staging manual and the future of TNM. Ann Surg Oncol. 2010;17:1471-4.

18. Montagna G, Sevilimedu V, Fornier M, Jhaveri K, Morrow M, Pilewskie ML. How effective is neoadjuvant endocrine therapy (NET) in downstaging the axilla and achieving breast-conserving surgery? Ann Surg Oncol. 2020;27:4702-10.

19. Weiss A, Wong S, Golshan M, et al. Patterns of axillary management in stages 2 and 3 hormone receptor-positive breast cancer by initial treatment approach. Ann Surg Oncol. 2019;26:4326-36.

20. Spring LM, Gupta A, Reynolds KL, et al. Neoadjuvant endocrine therapy for estrogen receptor-positive breast cancer: a systematic review and meta-analysis. JAMA Oncol. 2016;2:1477-86.

21. Kuehn T, Bauerfeind I, Fehm T, et al. Sentinel-lymph-node biopsy in patients with breast cancer before and after neoadjuvant chemotherapy (SENTINA): a prospective, multicentre cohort study. Lancet Oncol. 2013;14:609-18.

22. Haffty BG, McCall LM, Ballman KV, et al. Patterns of localregional management following neoadjuvant chemotherapy in breast cancer: results from ACOSOG Z1071 (Alliance). Int J Radiat Oncol Biol Phys. 2016;94:493-502.

23. Caudle AS, Yang WT, Krishnamurthy S, et al. Improved axillary evaluation following neoadjuvant therapy for patients with nodepositive breast cancer using selective evaluation of clipped nodes: implementation of targeted axillary dissection. J Clin Oncol. 2016;34:1072-8.

24. Consensus Guideline on the Management of the Axilla in Patients with Invasive/In Situ Breast Cancer. https://www.breastsurgeons. org/docs/statements/Consensus-Guideline-on-the-Management-o f-the-Axilla.pdf?v2. Accessed 20 Dec 2019.

25. Wagner LI, Gray RJ, Sparano JA, et al. Patient-reported cognitive impairment among women with early breast cancer randomly assigned to endocrine therapy alone versus chemoendocrine therapy: results from TAILORx. J Clin Oncol. 2020;38:1875-86.

26. Arima N, Nishimura R, Osako T, et al. Ki-67 index value and progesterone receptor status can predict prognosis and suitable treatment in node-negative breast cancer patients with estrogen receptor-positive and HER2-negative tumors. Oncol Lett. 2019;17:616-22.

27. Goto-Yamaguchi L, Yamamoto-Ibusuki M, Yamamoto Y, et al. Therapeutic predictors of neoadjuvant endocrine therapy response in estrogen receptor-positive breast cancer with reference to optimal gene expression profiling. Breast Cancer Res Treat. 2018;172:353-62.

28. Mackay A, Urruticoechea A, Dixon JM, et al. Molecular response to aromatase inhibitor treatment in primary breast cancer. Breast Cancer Res. 2007;9:R37.

29. Thornton MJ, Williamson HV, Westbrook KE, et al. Neoadjuvant endocrine therapy versus neoadjuvant chemotherapy in nodepositive invasive lobular carcinoma. Ann Surg Oncol. 2019;26:3166-77.

30. Feliciano Y, Mamtani A, Morrow M, Stempel MM, Patil S, Jochelson MS. Do calcifications seen on mammography after neoadjuvant chemotherapy for breast cancer always need to be excised? Ann Surg Oncol. 2017;24:1492-8.

31. Boileau JF, Poirier B, Basik M, et al. Sentinel node biopsy after neoadjuvant chemotherapy in biopsy-proven node-positive breast cancer: the SN FNAC study. J Clin Oncol. 2015;33:258-64.

32. Boughey JC, Suman VJ, Mittendorf EA, et al. Sentinel lymph node surgery after neoadjuvant chemotherapy in patients with node-positive breast cancer: the ACOSOG Z1071 (Alliance) clinical trial. JAMA. 2013;310:1455-61.

33. Chehade HEHH, El Tokhy O, Heeney J, Kasem A, Mokbel K. Is sentinel lymph node biopsy a viable alternative to complete axillary dissection following neoadjuvant chemotherapy in women with node-positive breast cancer at diagnosis? An updated meta-analysis involving 3398 patients. Am J Surg. 2016;212:969-81.

Publisher's Note Springer Nature remains neutral with regard to jurisdictional claims in published maps and institutional affiliations. 